\title{
In vitro activity of various combinations of antimicrobials against carbapenem-resistant Acinetobacter species in Singapore
}

\author{
Tze-Peng Lim ${ }^{1,4}$, Thean-Yen Tan $^{2}$, Winnie Lee ${ }^{1}$, Suranthran Sasikala ${ }^{2}$, Thuan-Tong Tan ${ }^{3}$, Li-Yang Hsu ${ }^{4}$ \\ and Andrea L Kwa ${ }^{1}$
}

Outbreaks of carbapenem-resistant Acinetobacter species have emerged, especially in Singapore. Combination therapy may be the only viable option until new antibiotics are available. The objective of this study was to identify potential antimicrobial combinations against carbapenem-resistant Acinetobacter baumannii and Acinetobacter species in Singapore. From an ongoing surveillance program, two isolates of $A$. baumannii and an isolate of Acinetobacter species that were multidrug resistant were selected on the basis of their unique resistance mechanisms. The two $A$. baumannii isolates carried the carbapenemase

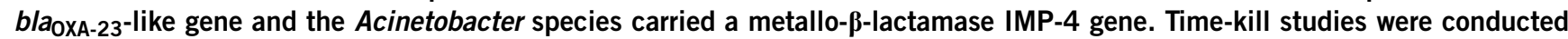
with approximately $10^{5} \mathrm{CFU} \mathrm{ml}^{-1}$ at baseline with 0.5 times minimum inhibitory concentrations (MICs) of polymyxin B and tigecycline, and at a maximally achievable clinical concentration of meropenem $\left(64 \mu \mathrm{gl}^{-1}\right)$ and rifampicin $\left(2 \mu \mathrm{g} \mathrm{ml}^{-1}\right)$, alone and in combinations. The MICs ( $\mu \mathrm{g} \mathrm{ml}^{-1}$ ) of Acinetobacter species A105, A. baumannii AB112 and A. baumannii AB8879 to polymyxin B/tigecycline/rifampicin/meropenem were found to be 1/0.5/4/64, 1/4/4/32 and 2/2/2/64, respectively. In time-kill studies, enhanced combined killing effects were observed in the tigecycline-rifampicin combination; the tigecycline-rifampicin and rifampicin-polymyxin B combination; and the rifampicin-polymyxin B combination for Acinetobacter species A105, A. baumannii AB112 and $A$. baumannii AB8879, respectively, with $>5$ log kill at $24 \mathrm{~h}$ suggesting synergism, with no regrowth observed at $72 \mathrm{~h}$. These findings demonstrate that in vitro synergy of antibiotic combinations in carbapenem-resistant Acinetobacter species may be strain dependent. It may guide us in choosing a preemptive therapy for carbapenem-resistant Acinetobacter species infections and warrants further investigations.

The Journal of Antibiotics (2009) 62, 675-679; doi:10.1038/ja.2009.99; published online 30 October 2009

Keywords: Acinetobacter species; carbapenem resistance; combination therapy

\section{INTRODUCTION}

Microbial resistance to antimicrobial agents is a serious problem that renders development of new treatment options an urgent priority. The alarming spread of antimicrobial resistance is threatening our therapeutic armamentarium. ${ }^{1,2}$ It is likely that effective treatment may not be available for many common infections in the near future, and we are at risk of returning to the preantibiotic era in the event of an outbreak. ${ }^{3}$ Broad-spectrum antimicrobial resistance in Gram-negative bacteria (for example, Acinetobacter baumannii and Pseudomonas aeruginosa) is especially worrisome and has worldwide implications.

Acinetobacter baumannii is an emerging Gram-negative bacillus associated with serious nosocomial infections; it is also associated with multiple mechanisms of resistance to various antimicrobial agents. ${ }^{4}$ Carbapenem resistance is now observed worldwide in A. baumannii, leading to limited therapeutic options. ${ }^{5}$ Several mechanisms are responsible for resistance to carbapenem in A. baumannii. These are reduced outer membrane permeability, penicillin-binding protein changes and carbapenamases. ${ }^{6}$ Treatment of multidrug-resistant Acinetobacter infections often represents a challenge to clinicians, ${ }^{7-9}$ and there are very few agents in the advanced stage of development designed to target multidrug-resistant Gram-negative bacteria. As a result, a task force from the Infectious Diseases Society of America (IDSA) has recently identified A. baumannii as a 'particularly problematic pathogen,' for which there is an urgent need for new and effective treatment strategies. ${ }^{10}$

Most of our carbapenem-resistant isolates were sensitive only to polymyxin/colistin. However, colistin treatment failures as a result of colistin resistance were reported. Furthermore, heterogeneous colistin resistance among multidrug-resistant isolates is now recognized in this region. ${ }^{11,12}$ Hence, the need for an effective combination antimicrobial

${ }^{1}$ Department of Pharmacy, Singapore General Hospital, Singapore; ${ }^{2}$ Department of Microbiology, Changi General Hospital, Singapore; ${ }^{3}$ Singapore General Hospital, National University of Singapore, Singapore and ${ }^{4}$ Department of Medicine, Yong Loo Lin School of Medicine, National University of Singapore, Singapore

Correspondence: Dr AL Kwa, Department of Pharmacy, Singapore General Hospital, Outram Road, Singapore 169609, Singapore.

E-mail: andrea.kwa.I.h@sgh.com.sg

Received 15 July 2009; revised 9 September 2009; accepted 18 September 2009; published online 30 October 2009 
therapy is urgent. The objective of this study was to identify potential antimicrobial combinations against carbapenem-resistant Acinetobacter species in Singapore. It is hoped that we can provide a robust assessment of the activity of different antimicrobial agents when used in combination and assist clinicians to efficiently identify potential antimicrobial combinations for such difficult-to-treat infections.

\section{MATERIALS AND METHODS}

\section{Species identification and OXA screening for test isolates}

Two clinical multidrug-resistant strains of A. baumannii (A. baumannii AB112 and A. baumannii AB8879) and an Acinetobacter species (Acinetobacter sp. A105) from isolates collected for surveillance were used in the time-kill studies. ${ }^{13}$ The three isolates were screened for blaOXA-23-like, blaOXA-24like, blaOXA-58-like and blaOXA-51-like genes using a multiplex PCR assay. Putative metallo- $\beta$-lactamase genes were amplified from the collection by using published degenerate primers. ${ }^{3,14-16}$ Selected PCR products were further sequenced to confirm their gene products. The ISAbal-OXA complex was detected with forward and reverse primers of the named genes, using the PCR protocol described by Turton et al. ${ }^{17}$ Another PCR-based multiplex assay was used to differentiate A. baumannii from other Acinetobacter species. ${ }^{18}$

\section{Minimum inhibitory concentration testing}

Minimum inhibitory concentrations (MICs) to a panel of antibiotics were obtained by commercial dehydrated microbroth dilution panels (Trek Diagnostics, East Grinstead, UK), performed according to the manufacturer's recommendations. MICs to rifampicin were obtained by a modified broth macrodilution method as described by the Clinical and Laboratory Standards Institute (CLSI). ${ }^{19}$ The studies were conducted in duplicate and were repeated at least once on a separate day.

\section{Antimicrobial agents}

Meropenem was obtained from Astra Zeneca (Dainippon Sumitomo Pharma Company Ltd, Oita, Japan). Polymyxin B and rifampicin were obtained from Sigma-Aldrich (St Louis, MO, USA). Tigecycline was obtained from Wyeth Pharmaceuticals (Pearl River, NY, USA). For polymyxin B and meropenem, a stock solution of each antimicrobial agent in sterile water was prepared, aliquoted and stored at $-70^{\circ} \mathrm{C}$. Tigecycline in solution was freshly prepared before each experiment. Before each susceptibility test, an aliquot of the drug was thawed and diluted to the desired concentrations with Cation-adjusted Mueller-Hinton broth (CAMHB). Conversely, rifampicin was dissolved in dimethyl sulfoxide and was then serially diluted to the desired final drug concentration. The final dimethyl sulfoxide concentration had no effect on Acinetobacter species and A. baumannii growth.

\section{Time-kill studies}

Time-kill studies were conducted with each antibiotic tested individually and in combination. For the purposes of our study, the maximum clinically achievable meropenem concentration of $64 \mathrm{\mu g} \mathrm{ml}^{-1}$, which represented a free peak concentration arising from a $2 \mathrm{~g}, 3 \mathrm{~h}$ infusion, was simulated. ${ }^{20}$ Rifampicin was tested at $2 \mu \mathrm{g} \mathrm{ml}^{-1}$, which represented a free peak concentration arising from a $600 \mathrm{mg}$, daily oral dose to maximize the use of the drug. ${ }^{21}$ The concentrations of polymyxin B and tigecycline were tested at $0.5 \times$ MIC to yield attainable experimental end points.

An overnight culture of the isolate was diluted into prewarmed CAMHB and incubated further at $35^{\circ} \mathrm{C}$ until log-phase growth was reached. The bacterial suspension was diluted with CAMHB according to absorbance at $630 \mathrm{~nm} ; 15 \mathrm{ml}$ of the suspension was transferred to $50-\mathrm{ml}$ sterile conical flasks, each containing $1 \mathrm{ml}$ of a drug dilution at 16 times the target concentration. The final concentration of the bacterial suspension in each flask was approximately $10^{5} \mathrm{CFU} \mathrm{ml}^{-1}$ (ranging from $1 \times 10^{5}$ to $5 \times 10^{5} \mathrm{CFU} \mathrm{ml}^{-1}$ ).

Flasks were incubated in a shaker water bath at $35^{\circ} \mathrm{C}$. Serial samples of broth were obtained from each flask at $0 \mathrm{~h}$ (baseline), and then at 2, 4, 8, 12 and $24 \mathrm{~h}$ after incubation. Samples were obtained in duplicate at each time period, except for the $24 \mathrm{~h}$ sample, which was tested in triplicate. Harvested broth samples $(0.5 \mathrm{ml})$ were first centrifuged at $10000 \times \mathrm{g}$ for $15 \mathrm{~min}$ and then reconstituted with sterile normal saline to their original volumes in order to minimize drug carryover. The total bacterial count for each sample was quantified by depositing serial 10 -fold dilutions of broth samples onto Mueller Hinton agar plates using a spiral-plater (Interscience, St Nom La Breteche, France). Inoculated plates were incubated in a humidified incubator $\left(35^{\circ} \mathrm{C}\right)$ for $18-24 \mathrm{~h}$, bacterial colonies were visually counted and the original bacterial density from the original sample was calculated on the basis of the dilution factor. Synergy was defined as $\geqslant 2 \log _{10}$ decrease in $\mathrm{CFU} \mathrm{ml}^{-1}$ for the antibiotic combination compared with its more active constituent, whereas additive effect was defined as $1 \log _{10}$ decrease in $\mathrm{CFU} \mathrm{ml}{ }^{-1}$ for the combination compared with its more active constituent at $24 \mathrm{~h}$. Bactericidal effect was defined as $\geqslant 3 \log _{10}$ decrease in $\mathrm{CFU} \mathrm{ml}^{-1}$, whereas antagonism was defined as the combination that yields colony counts higher than those detected with the more active single drug alone, at $24 \mathrm{~h}$.

\section{RESULTS}

\section{Susceptibility}

All isolates were resistant to meropenem, imipenem, ampicillin/ sulbactam, ciprofloxacin, gentamicin, piperacillin/tazobactam, cefepime and amikacin, but were susceptible to polymyxin B. There are no current CLSI susceptibility break points for rifampicin and tigecycline against Acinetobacter species. The MIC values are shown in Table 1.

\section{Resistance mechanisms}

Acinetobacter sp. A105 was positive for OXA-58 and IMP-type carbapenamases. A. baumannii $\mathrm{AB} 112$ and A. baumannii AB8879 were positive for OXA-23 and OXA-51 $\beta$-lactamases. ${ }^{22}$

\section{Time-kill studies}

Figures 1a, 2a and 3a show the microbiological responses observed in single-drug time-kill studies. The number of $\mathrm{CFU} \mathrm{ml}{ }^{-1}$ over time in response to the tested antibiotic(s) depicts the microbiological response. Polymyxin B was the only single antibiotic to demonstrate consistent bactericidal activity against all three test isolates. The second most active single antibiotic was meropenem, in two out of the three test strains. However, isolate regrowth was observed for all single antibiotics by $24 \mathrm{~h}$.

For test strain Acinetobacter sp. A105, all antibiotic combinations showed rapid bactericidal activity within $2 \mathrm{~h}$ of initial inoculation. Bacterial counts fell below the lower threshold of detection within $2 \mathrm{~h}$ for tigecycline-rifampicin, meropenem-rifampicin and polymyxin B-meropenem antibiotic combinations, and tigecycline-rifampicin combination remained so throughout the 24-h testing period. These three combinations fulfilled the microbiological definition of

Table 1 Minimum inhibitory concentration $\left(\mu \mathrm{g} \mathrm{ml}^{-1}\right.$ ) results against the two $A$. baumannii isolates and one Acinetobacter species

\begin{tabular}{|c|c|c|c|}
\hline Antimicrobial & $\begin{array}{l}\text { Acinetobacter } \\
\text { species A105 }\end{array}$ & $\begin{array}{c}\text { A. baumannii } \\
\text { AB112 }\end{array}$ & $\begin{array}{c}\text { A. baumannii } \\
\text { AB8879 }\end{array}$ \\
\hline Meropenem & 64 & 32 & 64 \\
\hline Polymyxin B & 1 & 1 & 2 \\
\hline Rifampicin & 4 & 4 & 2 \\
\hline Tigecycline & 0.5 & 4 & 2 \\
\hline Ampicillin-sulbactam & $\geqslant 128$ & 16 & $\geqslant 128$ \\
\hline Ciprofloxacin & $\geqslant 16$ & $\geqslant 16$ & $\geqslant 16$ \\
\hline Gentamicin & $\geqslant 64$ & $\geqslant 64$ & $\geqslant 64$ \\
\hline Minocycline & $\leqslant 0.25$ & 1 & 4 \\
\hline Piperacillin-tazobactam & $\geqslant 256$ & $\geqslant 256$ & $\geqslant 256$ \\
\hline Cefepime & $\geqslant 64$ & $\geqslant 64$ & $\geqslant 64$ \\
\hline Amikacin & $\geqslant 128$ & $\geqslant 128$ & $\geqslant 128$ \\
\hline Ceftazidime & $\geqslant 128$ & $\geqslant 128$ & $\geqslant 128$ \\
\hline
\end{tabular}



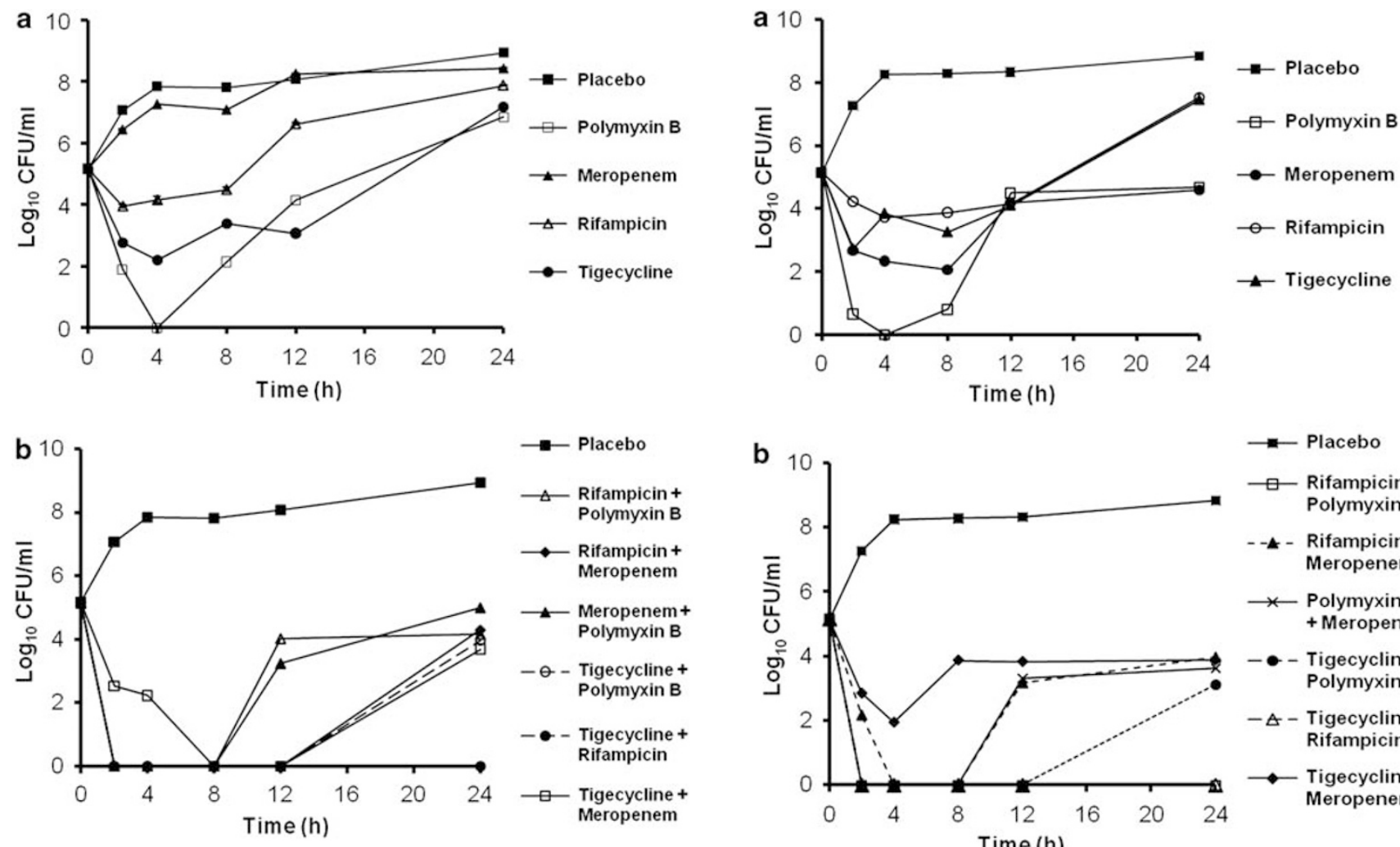

Figure 1 Microbiological responses observed in Acinetobacter sp. A105: single-drug system (a), two antimicrobial combinations (b). The number of CFU ml ${ }^{-1}$ over time in response to the tested antibiotic(s) depicts microbiological responses. A considerable reduction ( $>99 \%$ ) in bacterial burden was observed at $24 \mathrm{~h}$ for the tigecycline-rifampicin combination.

antibiotic synergy for all the tested time periods. Synergistic activity was intermittently present for the other antibiotic combinations during the 24-h testing time period, but bacterial regrowth was observed at the 24-h end point.

For test strain A. baumannii AB112, all antibiotic combinations other than meropenem-tigecycline rapidly achieved a $>3 \log _{10}$ reduction in bacterial counts within the first $4 \mathrm{~h}$ of testing. Only the two most effective bactericidal combinations of tigecycline-rifampicin and rifampicin-polymyxin B showed synergistic activity at the end of the 24-h testing period. The least-effective combination for this isolate was meropenem-rifampicin, with only marginally increased activity compared with meropenem alone.

For test strain A. baumannii AB8879, all antibiotic combinations with polymyxin $B$ achieved bactericidal and synergistic activity in the first $4 \mathrm{~h}$ of testing, but only the rifampicin-polymyxin B combination maintained synergistic activity at $24 \mathrm{~h}$. The least-effective combination for this isolate was meropenem-rifampicin, with only marginal improvement for the combination when compared with the activity of meropenem alone.

\section{DISCUSSION}

Few treatment options remain for serious infections caused by multidrug-resistant and carbapenem-resistant $A$. baumannii. Combination therapy (in view of potential synergistic activity) for multidrugresistant Gram-negative bacteria may be more effective than monotherapy, ${ }^{23}$ and may allow the use of antibiotics with marginal activity against the target organism.

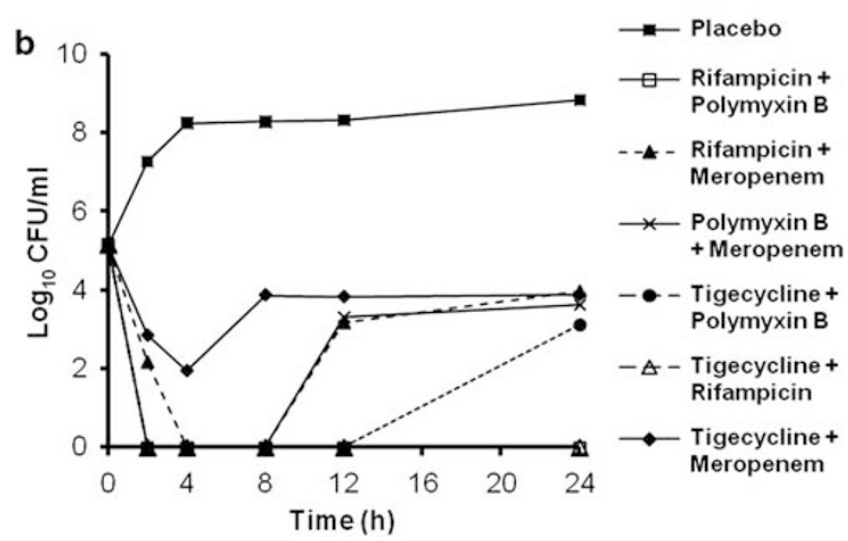

Figure 2 Microbiological responses observed in A. baumannii AB112: single-drug system (a), two antimicrobial combinations (b). The number of CFU ml ${ }^{-1}$ over time in response to the tested antibiotic(s) depicts microbiological responses. A considerable reduction ( $>99 \%)$ in bacterial burden was observed at $24 \mathrm{~h}$ for tigecycline-rifampicin and rifampicinpolymyxin B combinations.

Carbapenem-hydrolyzing OXA enzymes are the most important cause of carbapenem resistance in A. baumannii worldwide. Although these oxacillinases are weaker hydrolyzers of carbapenems in vitro than are metallo- $\beta$-lactamase, the presence of the promoter sequence, ISAbal, can result in clinically significant resistance to carbapenems. A. baumannii carrying OXA-23 is now found in most parts of the world and are often responsible for many outbreaks. ${ }^{6}$ Previous investigations revealed that the most common carbapenemase gene responsible in carbapenem-resistant isolates in our institutions was $b l a_{\text {oxa-23 }}$ and not $b l a_{\text {oxa-51 }} \cdot{ }^{22,24}$ The two tested A. baumannii strains in this study carried both the $b l a_{\mathrm{OXA}-23}$-like and $b l a_{\mathrm{OXA}-51}$-like carbapenemase genes, and both isolates possessed ISAbal upstream of the $b l a_{\text {OXA-23 }}$ gene (results not shown). A. sp. A 105 (with $b l a_{\text {OXA-58-like }}$

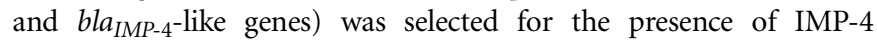
metallo- $\beta$-lactamase, as metallo- $\beta$-lactamase genes are increasingly being reported. ${ }^{25,26}$ It is likely that as multidrug-resistant isolates become more common, we will need to define and target in our selection of antibiotics a combination based on a library of data such as those presented here on various combinations that are useful in strains with different resistance determinants.

In this study, clinically achievable concentrations of the tested antibiotics were used. For example, previous studies have shown that extending the infusion duration of meropenem from $30 \mathrm{~min}$ to $3 \mathrm{~h}$ increases the probability of bactericidal target attainment. ${ }^{20,27}$ Despite this, synergistic activity between meropenem and other antibiotic combinations was only observed in one out of three strains. 

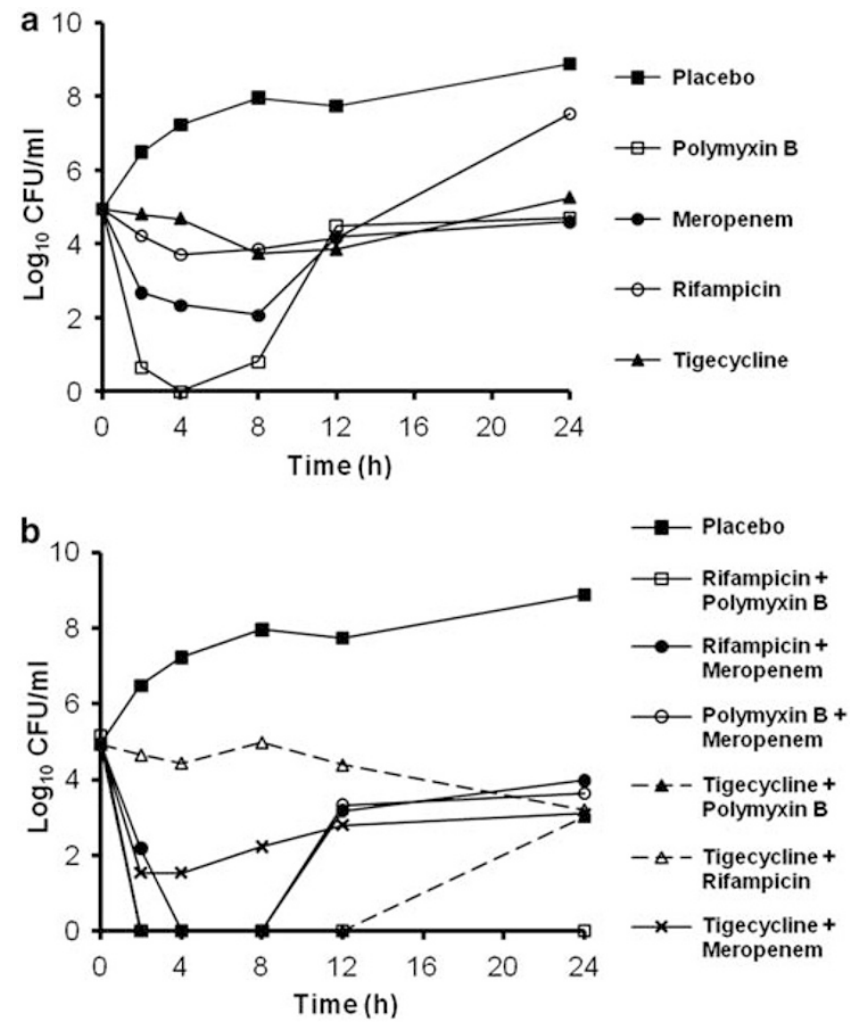

Figure 3 Microbiological responses observed in A. baumannii AB8879: single-drug system (a), two antimicrobial combinations (b). The number of CFU ml-1 over time in response to the tested antibiotic(s) depicts microbiological responses. A considerable reduction (>99\%) in bacterial burden was observed at $24 \mathrm{~h}$ for the rifampicin-polymyxin B combination.

There was no antibiotic combination that reliably demonstrated synergistic activity against all isolates, although rifampicin-polymyxin and tigecycline-rifampicin combinations were bactericidal and synergistic for two of the three isolates, respectively.

Time-kill studies are labor intensive for routine use and will not provide results in a clinically relevant time frame. However, screening for useful antibiotic combinations in a local population of antibiotic-resistant Acinetobacter species with well-defined resistance mechanisms may allow the empirical selection of combination antibiotic therapy, where clinically indicated. Clearly, more quantitative information regarding such synergistic and antagonistic relationships is both valuable and necessary for evaluating the effectiveness of various antimicrobial agent combinations. Other potential models that have been used to determine synergistic activity include an in vitro pharmacodynamic infection model in which human-like (fluctuating) drug concentration profiles are simulated, and chequer-board titrations, Etest and in vivo animal testing are carried out. However, it is worth noting that the correlation between each model and actual clinical outcomes remains to be clearly elucidated. For example, studies have found conflicting results for the same antimicrobial combinations comparing animal in vivo studies with a follow-up clinical pilot study. ${ }^{28,29}$

In this study, we performed time-kill analysis only for three different Acinetobacter species with similar MICs against various antimicrobial agents. The differences in the mechanisms of resistance seemed to result in different effective antibiotic combinations. Hence, one effective antimicrobial combination for one isolate cannot be assumed to be effective for another isolate of the same species. Either combination testings are carried out for every carbapenem-resistant isolates or lengthy experiments are conducted to determine phenotypic response to different combinations of resistance genes. Neither of the two methods mentioned are technically easy nor practically feasible, but may prove necessary under pressure of the spread of such bacteria.

In A. baumannii, the AdeABC efflux pump, a member of the resistance-nodulation-cell-division family, has been well characterized. Aminoglicosides, tetracyclines, erythromycin, chloramphenicol, trimethoprim, fluoroquinolones, some $\beta$-lactams and also recently tigecycline were found to be substrates for this pump. Drugs, as substrates for the AdeABC pump, can increase the expression of AdeABC genes, leading to multidrug resistance. ${ }^{30}$ Although AdeABC multidrug efflux pumps are intrinsic to A. baumannii, and the AdeDE and AdeXY pumps are found predominantly in Acinetobacter genomospecies $3,^{31}$ we did not have any additional data to confirm the overexpression of these efflux genes, which can contribute to multidrug resistance in our isolates. Hence, with the knowledge of different carbapenemase genes (which confer expression of different types of carbapenemases) in our isolates, we can only prostulate why carbapenem as a part of combination antibiotics does not work in synergism, whereas combinations using non- $\beta$-lactams work better against our isolates.

\section{CONCLUSION}

Various antibiotic combinations against carbapenem-resistant Acinetobacter species were tested and reported with varied efficacies. Thus, it is clear that antibacterial effects can differ according to resistance mechanisms. ${ }^{32}$

We selected strains that had mechanisms common in our settings, according to our surveillance program. For example, A. baumannii AB8879 was an outbreak strain in our burns unit. We propose that combination testings be made a part of molecular mechanisms surveillance programs to be effective. This study demonstrated the utility of synergy testing in a selection of multidrug-resistant Acinetobacter species to determine the activity of specific antibiotic combinations. This may facilitate the optimal use of antimicrobial agents by guiding a rational selection of future antibiotic combinations for therapy.

\section{CONFLICT OF INTEREST}

The authors declare no conflict of interest.

\section{ACKNOWLEDGEMENTS}

This study was supported in part by National Medical Research Council and SingHealth Foundation.

1 Gold, H. S. \& Moellering, R. C. Jr. Antimicrobial-drug resistance. N. Engl. J. Med. 335, 1445-1453 (1996).

2 Neu, H. C. The crisis in antibiotic resistance. Science 257, 1064-1073 (1992).

3 Landman, D. et al. Citywide clonal outbreak of multiresistant Acinetobacter baumannii and Pseudomonas aeruginosa in Brooklyn, NY: the preantibiotic era has returned. Arch. Intern. Med. 162, 1515-1520 (2002).

4 Bonomo, R. A. \& Szabo, D. Mechanisms of multidrug resistance in Acinetobacter species and Pseudomonas aeruginosa. Clin. Infect. Dis. 43(Suppl 2), S49-S56 (2006).

5 Peleg, A. Y., Seifert, H. \& Paterson, D. L. Acinetobacter baumannii: emergence of a successful pathogen. Clin. Microbiol. Rev. 21, 538-582 (2008).

6 Poirel, L. \& Nordmann, P. Carbapenem resistance in Acinetobacter baumannii: mechanisms and epidemiology. Clin. Microbiol. Infect. 12, 826-836 (2006).

7 Maragakis, L. L. \& Perl, T. M. Acinetobacter baumannii: epidemiology, antimicrobial resistance, and treatment options. Clin. Infect. Dis. 46, 1254-1263 (2008). 
8 Levin, A. S. et al. Intravenous colistin as therapy for nosocomial infections caused by multidrug-resistant Pseudomonas aeruginosa and Acinetobacter baumannii. Clin. Infect. Dis. 28, 1008-1011 (1999).

9 Centers for Disease Control and Prevention (CDC). Acinetobacter baumannii infections among patients at military medical facilities treating injured U.S. service members, 2002-2004. MMWR Morb. Mortal. Wkly. Rep. 53, 1063-1066 (2004).

10 Talbot, G. H. et al. Bad bugs need drugs: an update on the development pipeline from the Antimicrobial Availability Task Force of the Infectious Diseases Society of America. Clin. Infect. Dis. 42, 657-668 (2006).

$11 \mathrm{Li}$, J. et al. Heteroresistance to colistin in multidrug-resistant Acinetobacter baumannii. Antimicrob. Agents. Chemother. 50, 2946-2950 (2006).

$12 \mathrm{Yau}, \mathrm{W}$. et al. Colistin hetero-resistance in multidrug-resistant Acinetobacter baumannii clinical isolates from the Western Pacific region in the SENTRY antimicrobial surveillance programme. J. Infect. 58, 138-144 (2009).

$13 \mathrm{Hsu}, \mathrm{L}$. Y. et al. Antimicrobial drug resistance in Singapore hospitals. Emerg. Infect. Dis. 13, 1944-1947 (2007).

14 Woodford, N. et al. Multiplex PCR for genes encoding prevalent OXA carbapenemases in Acinetobacter spp. Int. J. Antimicrob. Agents 27, 351-353 (2006).

15 Brown, S., Young, H. K. \& Amyes, S. G. Characterisation of OXA-51, a novel class D carbapenemase found in genetically unrelated clinical strains of Acinetobacter baumannii from Argentina. Clin. Microbiol. Infect. 11, 15-23 (2005).

16 Nordmann, P. \& Poirel, L. Emerging carbapenemases in Gram-negative aerobes. Clin. Microbiol. Infect. 8, 321-331 (2002).

17 Turton, J. F. et al. The role of ISAba1 in expression of OXA carbapenemase genes in Acinetobacter baumannii. FEMS Microbiol. Lett. 258, 72-77 (2006).

18 Chen, T. L. et al. Comparison of one-tube multiplex PCR, automated ribotyping and intergenic spacer (ITS) sequencing for rapid identification of Acinetobacter baumannii. Clin. Microbiol. Infect. 13, 801-806 (2007).

19 Clinical and Laboratory Standards Institute. Performance Standards for Antimicrobial Testing: Seventeenth Informational Supplement M100-S17(CLSI: Wayne, Pennsylvania, USA, 2007)

20 Jaruratanasirikul, S., Sriwiriyajan, S. \& Punyo, J. Comparison of the pharmacodynamics of meropenem in patients with ventilator-associated pneumonia following administration by 3-h infusion or bolus injection. Antimicrob. Agents. Chemother. 49, 1337-1339 (2005)
21 Gumbo, T. et al. Concentration-dependent mycobacterium tuberculosis killing and prevention of resistance by rifampin. Antimicrob. Agents. Chemother. 51, 3781-3788 (2007).

22 Tan, T. T., Widjaya, L., Hsu, L. Y. \& Koh, T. H. Characterization of resistance genes among Acinetobacter spp. isolates in Singaporean Hospitals. 18th European Congress of Clinical Microbiology and Infectious Diseases. Barcelona Spain, 2008.

23 Urban, C., Segal-Maurer, S. \& Rahal, J. J. Considerations in control and treatment of nosocomial infections due to multidrug-resistant Acinetobacter baumannii. Clin. Infect. Dis. 36, 1268-1274 (2003).

24 Koh, T. H., Sng, L. H., Wang, G. C., Hsu, L. Y. \& Zhao, Y. IMP-4 and OXA betalactamases in Acinetobacter baumannii from Singapore. J. Antimicrob. Chemother. 59, 627-632 (2007).

25 Tognim, M. C., Gales, A. C., Penteado, A. P., Silbert, S. \& Sader, H. S. Dissemination of IMP-1 metallo-beta-lactamase-producing Acinetobacter species in a Brazilian teaching hospital. Infect. Control. Hosp. Epidemiol. 27, 742-747 (2006).

26 Wang, H. et al. Molecular epidemiology of clinical isolates of carbapenem-resistant Acinetobacter spp. from Chinese hospitals. Antimicrob. Agents. Chemother. 51, 4022-4028 (2007).

27 Li, C., Kuti, J. L., Nightingale, C. H. \& Nicolau, D. P. Population pharmacokinetic analysis and dosing regimen optimization of meropenem in adult patients. J. Clin. Pharmacol. 46, 1171-1178 (2006).

28 Montero, A. et al. Antibiotic combinations for serious infections caused by carbapenemresistant Acinetobacter baumannii in a mouse pneumonia model. J. Antimicrob. Chemother. 54, 1085-1091 (2004).

29 Saballs, M. et al. Rifampicin/imipenem combination in the treatment of carbapenemresistant Acinetobacter baumannii infections. J. Antimicrob. Chemother. 58, 697-700 (2006).

30 Wieczorek, P. et al. Multidrug resistant Acinetobacter baumannii-the role of AdeABC (RND family) efflux pump in resistance to antibiotics. Folia. Histochem. Cytobiol. 46, 257-267 (2008).

31 Koh, T. H., Sng, L. H., Wang, G. C., Hsu, L. Y. \& Zhao, Y. Carbapenemase and efflux pump genes in Acinetobacter calcoaceticus-Acinetobacter baumannii complex strains from Singapore. J. Antimicrob. Chemother. 60, 1173-1174 (2007).

32 Song, J. Y. et al. In vitro activities of carbapenem/sulbactam combination, colistin, colistin/rifampicin combination and tigecycline against carbapenemresistant Acinetobacter baumannii. J. Antimicrob. Chemother. 60, 317-322 (2007) 